\title{
Astilbin emulsion improves guinea pig lesions in a psoriasis-like model by suppressing IL-6 and IL-22 via p38 MAPK
}

\author{
JINGHONG YU ${ }^{1}$, ZHICAI XIAO ${ }^{1}$, RUIZHI ZHAO ${ }^{1}$, CHUANJIAN LU $^{2}$ and YUEMEI ZHANG ${ }^{1}$ \\ ${ }^{1}$ Department of Chinese Medicine Property Team, The Second Affiliated Hospital, Guangzhou University of \\ Chinese Medicine, Guangzhou, Guangdong 510006; ${ }^{2}$ Guangdong Province Key Laboratory of \\ Clinical Research on Traditional Chinese Medicine Syndrome, Guangzhou, Guangdong 510115, P.R. China
}

Received January 25, 2017; Accepted August 31, 2017

DOI: $10.3892 / \mathrm{mmr} .2017 .8343$

\begin{abstract}
Astilbin has anti-inflammatory and immunoregulatory effects, and is frequently used in prescriptions treating psoriasis; however, the mechanism remains to be fully elucidated. In the present study, the effect of an astilbin microemulsion on a psoriasis-like model in guinea pigs was examined, and the underlying mechanism was investigated. The levels of interkeukin (IL)-6, IL-17A and IL-22 were determined using fluorescent reverse transcription-quantitative polymerase chain reaction analysis and enzyme-linked immunosorbent assays. The phosphorylation of p38 and extracellular signal-regulated kinase (ERK)1/2 was detected using western blot analysis. Compared with the untreated control, astilbin significantly ameliorated the lesions induced by propranolol hydrochloride. The effect of astilbin on cytokine levels were cytokine- and drug-concentration-dependent. At a concentration of $2.22 \mu \mathrm{M}$, astilbin decreased the mRNA expression levels of IL-6, IL-17A and IL-22 in lipopolysaccharide (LPS)-induced HaCaT cells by 89,69.1 and 69.3\%, respectively. However, $2.22 \mu \mathrm{M}$ astilbin had no effect on the protein expression of IL-17A, and decreased the protein expression levels of IL-6 and IL-22 by 79.2 and $49.5 \%$, respectively $(\mathrm{P}<0.05)$. At a concentration of $11.10 \mu \mathrm{M}$, astilbin decreased the mRNA expression of IL-6, which was significantly induced by LPS, and significantly $(\mathrm{P}<0.05)$ decreased the protein expression levels of IL-6 and IL-22. Additionally, astilbin inhibited the LPS-induced activation of phosphorylated p38. These results suggested that astilbin has the potential to be developed into a topical drug for the treatment of psoriasis via the inhibition of inflammatory cytokines.
\end{abstract}

Correspondence to: Professor Ruizhi Zhao, Department of Chinese Medicine Property Team, The Second Affiliated Hospital, Guangzhou University of Chinese Medicine, 55 Neihuanxi Road, Guangzhou, Guangdong 510006, P.R. China

E-mail: 13610241754@163.com

Key words: astilbin, psoriasis, interleukin-6, interleukin-22, p38 mitogen-activated protein kinase, keratinocyte

\section{Introduction}

Psoriasis is an immune-mediated autoimmune skin disorder, which is characterized histologically by inflammatory cell infiltrate, hyperproliferative keratinocytes in the epidermis and hyperplasia of the dermal papilla, and affects $1-3 \%$ of the population worldwide (1). Several methods are used to treat psoriasis, including ultraviolet treatment, topical or systemic drugs and biological therapy. However, the limited long-term effectiveness, severe side effects, high cost and frequency of relapse associated with these treatments, and satisfactory treatment in only $27 \%$ of patients, novel therapies are required (2).

Astilbin, 3,3',4',5,7-pentahydroxyflavanone-3-[6-deoxy(1-mannopyranoside)], the structure of which is shown in Fig. 1, exists widely in herbs, including Smilax glabra Roxb (3), Dimorphandra nollis (4), Engelhardis roxburghiana (5) and Astilbe chinensis (6). Astilbin has a wide range of pharmacological effects, including inhibiting the adhesion of T lymphocytes (7), effects against experimental diabetic nephropathy (8), inhibiting contact hypersensitivity (9), inducing immunosuppression (10), and decreasing functionally activated T and B cells (11). All of these properties are associated with the mechanisms controlling psoriasis.

Therefore, in the present study, the effect of astilbin emulsion on a propranolol hydrochloride-induced guinea pig model of psoriasis was investigated, and the underlying mechanism was examined in an immortalized human keratinocyte (HaCaT) cell line stimulated by lipopolysaccharide (LPS). The results showed that astilbin had anti-psoriatic effects, and the mechanism involved inhibiting p38 mitogen-activated protein kinase (MAPK), with subsequent inhibition of interleukin (IL)-6 and IL-22.

\section{Materials and methods}

Drugs and reagents. Astilbin (purity $>98 \%$ ) was purchased from Purui Technology Company (Chengdu, China). Propranolol chloride (purity $>98 \%$ ) was purchased from Maiyuan Chemical Reagent Co. (Wuhan, China), and the $9.5 \%$ astilbin microemulsion and $5 \%$ propranolol emulsion were prepared at the Pharmaceutical Laboratory of Guangdong Hospital of Chinese Medicine (Guangzhou, China). RPMI-1640 medium, 
fetal bovine serum (FBS) and streptomycin-penicillin were purchased from Thermo Fisher Scientific, Inc. (Waltham, MA, USA). TRIzol was purchased from Takara Biotechnology Co., Ltd. (Dalian, China). Methyl thiazolyl tetrazolium (MTT) and LPS were purchased from Sigma-Aldrich; Merck Millipore (Darmstadt, Germany). The reverse transcription kit and SYBR-Green/Fluorescein qPCR Master mix were purchased from Thermo Fisher Scientific, Inc. (Carlsbad, CA, USA). The mRNA primers for human $\beta$-actin, IL-6, IL-17 and IL-22 were synthesized by Thermo Fisher Scientific, Inc. (Shanghai, China). The IL-6, IL-17A and IL-22 enzyme-linked immunosorbent assay (ELISA) kits and the ELISA buffer kit were purchased from PeproTech EC, Ltd. (London, UK). The bicinchoninic acid protein assay kit was purchased from Thermo Fisher Scientific, Inc. The Clarity ${ }^{\mathrm{TM}}$ Western ECL substrate and Precision Plus Protein Kaleidoscope were purchased from Bio-Rad Laboratories, Inc. (Hercules, CA, USA). RIPA buffer (cat. no. 9806S), p38 (cat. no. 8690S), phosphorylated p38 (cat. no. 9211S), extracellular signal-regulated kinase (ERK)1/2 (cat. no. 4695S), phosphorylated ERK1/2 (cat. no. 4370S) and GAPDH (cat. no. 2118S) antibodies, and anti-rabbit IgG, horseradish peroxidase-conjugated antibody (cat. no. 7074S) were purchased from Cell Signaling Technology, Inc. (Danvers, MA, USA).

Animals and cell lines. Male albino guinea pigs, weighing 250-300 g, were purchased from the Medical Laboratory Animal Center of Guangdong (Guangzhou, China) at 4 weeks of age. All animal experiments were performed according to the NIH guidelines for the Care and Use of Laboratory Animals, and were approved by the Animal and Ethics Review Committee of Guangzhou University of Chinese Medicine (protocol approval no. 2011001) on 24th December 2010.

The $\mathrm{HaCaT}$ cell line was purchased from the China Centre for Type Culture Collection (Wuhan, China). The cells were cultured in RPMI-1640 supplemented with 5\% FBS, $10 \mu \mathrm{g} / \mathrm{ml}$ of streptomycin and $10 \mathrm{U} / \mathrm{ml}$ of penicillin, and were incubated in a humidified atmosphere with $5 \% \mathrm{CO}_{2}$ and $95 \%$ air at $37^{\circ} \mathrm{C}$.

In vivo experiments. The animals were housed in a $24^{\circ} \mathrm{C}$ humidity-controlled room with 12-h light-dark cycle and allowed free access to standard diet and tap water. following acclimatization for 1 week, the guinea pigs were randomly divided into a control group $(n=6)$ and model group $(n=12)$. The ears of the animals were shaved in the model group, and a $5 \%$ propranolol emulsion $(0.2 \mathrm{ml} / \mathrm{ear})$ was administered topically three times a day to induce psoriasis-like lesions. After 8 days, the model was established (12).

The animals in model group were then separated into a model control $(n=6)$ and experimental $(n=6)$ group. A 9.5\% microemulsion of astilbin was administered topically to animals in the experimental group at a dose of $0.2 \mathrm{~g} /$ ear once per day, whereas the animals in the model control group were topically administered with the same quantity of blank emulsion. After 1 week, all guinea pigs were sacrificed and the ears were cut for the preparation of paraffin sections with thickness of $\sim 5 \mu \mathrm{m}$, which were stained using hematoxylin and eosin for histopathological evaluations using the Olympus microscope CX31 (Olympus Corporation, Tokyo, Japan), Baker scores were assigned (13) according to the criteria shown in Table I.

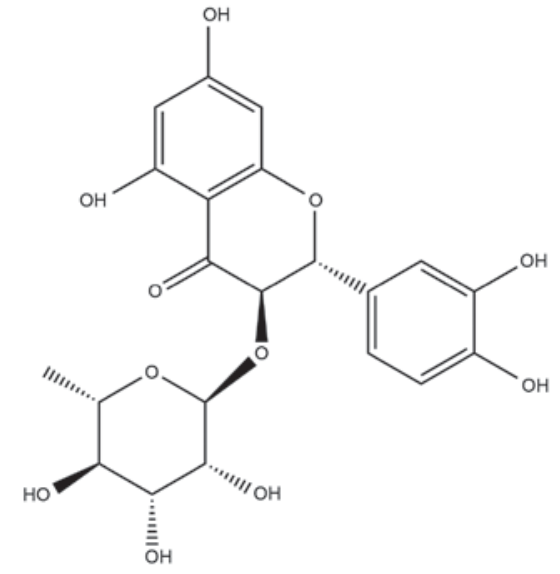

Figure 1. Structure of astilbin.

Table I. Baker score criteria.

\begin{tabular}{llc}
\hline Region & \multicolumn{1}{c}{ Pathological change } & Score \\
\hline Corneous layer & Munro abscess & 2.0 \\
& Hyperkeratosis & 0.5 \\
& Parakeratosis & 1.0 \\
Epidermis & Lack or attenuation of granular layer & 1.0 \\
& Acanthosis & 1.0 \\
& Elongation of the rete ridges & $0.5-1.5$ \\
Dermis & Lymphocytic infiltrate & $0.5-2.0$ \\
& Papillary papillae congestion & 0.5 \\
& Telangiectasia of superficial derma & 0.5 \\
\hline
\end{tabular}

Cell growth assay. A 5.0-mg quantity of astilbin (>98\% in purity) was precisely weighed and dissolved into $50 \mu \mathrm{l}$ dimethyl sulfoxide as a stock solution $(222 \mathrm{mM})$. Prior to experiments, the stock solution of astilbin was diluted in PBS and then prepared to the experimental doses.

The growth inhibition induced by astilbin in $\mathrm{HaCaT}$ cells was assessed using an MTT assay. The cells were seeded in a 96-well plate at a density of 2,000 cells/well and cultured for $24 \mathrm{~h}$ to allow the cells to adhere to the plates. Subsequently, the cells were exposed to the presence or absence of different concentrations of astilbin, $(6.66,13.88,27.75,55.50$ and $222.00 \mu \mathrm{M})$ in RPMI-1640 medium. The plates were placed at $37^{\circ} \mathrm{C}$ for $24 \mathrm{~h}$, following which $20 \mu \mathrm{l}$ MTT $(5 \mathrm{mg} / \mathrm{ml})$ was added to each well of cells, and the reaction was allowed to proceed at $37^{\circ} \mathrm{C}$ for $4 \mathrm{~h}$ in the dark. The supernatant was then collected and the cells were washed with PBS. The formazan salts produced were dissolved with dimethyl sulfoxide, and the absorbance was measured at $570 \mathrm{~nm}$ using a Vector 5 multifunctional immune analyzer (PerkinElmer, Inc., Waltham, MA, USA).

Reverse transcription-quantitative polymerase chain reaction (RT-qPCR) analysis of the mRNA expression levels of IL-6, $I L-17$ and $I L-22$. Following pretreatment with astilbin at different concentrations $(2.22$ and $11.10 \mu \mathrm{M})$ for $24 \mathrm{~h}$ and subsequent treatment with LPS $(5 \mu \mathrm{g} / \mathrm{ml})$ for $2 \mathrm{~h}$, total 
Table II. Primer sets used for reverse transcription-quantitative polymerase chain reactions.

\begin{tabular}{lll}
\hline Gene & Forward primer $\left(5^{\prime}-3^{\prime}\right)$ & Reverse primer $\left(5^{\prime}-3^{\prime}\right)$ \\
\hline$\beta$-actin & CGGGAAATCGTGCGTGACAT & CAGGAAGCAAGGCTGGAAGA \\
IL-6 & CTGAGGGCTCTTCGGCAAAT & GCCCAGTGGACAGGTTTCTG \\
IL-17A & GCCTTCAAGACTGAACACCG & TGACATGCCATTCCTCAGGG \\
IL-22 & AACTAACCCCCTTTCCCTGC & AACGCAGGGGTTCATTTGGA
\end{tabular}

IL, interleukin.

RNA from the HaCaT cells in 6-well plates was isolated using TRIzol reagent. cDNA was generated from $1 \mu \mathrm{g}$ total RNA and reverse transcriptase using the First Strand cDNA synthesis kit on a 7500 Fast Real-Time PCR system (ABI; Thermo Fisher Scientific, Inc.). The specific primer pairs are shown in Table II.

The reaction products were detected by measuring the binding of SYBR Green I to DNA using SYBR Green PCR Master mix (Thermo Fisher Scientific, Inc.). Optimization of the amplification reaction was assured through dissociation curve analysis. The basic protocol for the RT-qPCR procedure was an initial incubation step at $95^{\circ} \mathrm{C}$ for $7 \mathrm{~min}$, followed by 45 cycles of $95^{\circ} \mathrm{C}$ for $10 \mathrm{sec}$ and $60^{\circ} \mathrm{C}$ for $30 \mathrm{sec}$. All experiments were run in triplicate, and the relative expression values were normalized to the expression value of $\beta$-actin, followed by analysis using the $2^{-\Delta \Delta \mathrm{Cq}}$ method (14).

ELISA for IL-6, IL-17A and IL-22. Following pretreatment with astilbin at different concentrations $(2.22$ and $11.10 \mu \mathrm{M})$ for $24 \mathrm{~h}$ and subsequent treatment with LPS $(5 \mu \mathrm{g} / \mathrm{ml})$ for $2 \mathrm{~h}$, the incubation media of the $\mathrm{HaCaT}$ cells was collected and centrifuged at $3,000 \times \mathrm{g}$ for $3 \mathrm{~min}$ at room temperature to remove floating cells. The clear supernatant was collected to perform ELISA analyses, according to the manufacturer's protocol, to assess the expression levels of IL-6, IL-17A and IL-22. The 96-well plates loaded with the samples were read at $450 \mathrm{~nm}$ on a Vector 5 multifunctional immune analyzer. The levels of human IL-6, IL-17 and IL-22 in each well calculated as $\mathrm{pg} / \mathrm{ml}$.

Western blot analysis of protein levels of phosphorylated p38 $M A P K$ and phosphorylated ERK1/2. The HaCaT cells were washed twice with ice-cold PBS and lysed in ice-cold RIPA lysis buffer containing $1 \%$ proteinase inhibitors and $1 \%$ phosphatase inhibitors. The lysates were centrifuged at 20,800 x g for $15 \mathrm{~min}$ at $4^{\circ} \mathrm{C}$ to obtain the total protein. The protein concentrations were determined using the bicinchoninic acid protein assay kit, and cell lysates containing $30 \mu \mathrm{g}$ total proteins were separated on a $12 \%$ SDS-PAGE and transferred electrophoretically onto polyvinylidene fluoride membranes. Following incubation in blocking solution (5\% non-fat milk solution in Tris-buffered saline with Tween-20) for $1 \mathrm{~h}$ at room temperature, the membranes were incubated with 1:1,000 dilutions of primary antibodies against p38 MAPK, ERK1/2, phosphorylated ERK1/2 or GAPDH overnight at $4^{\circ} \mathrm{C}$. GAPDH was used as control. The membranes were then washed three times using Tris- $\mathrm{HCl}$ with $20 \%$ Tween- 80 and incubated with a 1:2,000 dilution of secondary antibody for $1 \mathrm{~h}$ at $4^{\circ} \mathrm{C}$. Finally, the membranes were washed three more times. Protein expression on the membranes was visualized using an enhanced chemiluminescence detection system. Densitometric analysis was performed using Quantity One software (version 4.6.2; Bio-Rad Laboratories, Inc.) to scan the signals. The signal quantities shown were standardized to the background and normalized for loading. The controls were set as 1.0, and the fold-changes were plotted in relation to the control value. The results represent the average values of three independent experiments.

Statistical analysis. All data are expressed as the mean \pm standard deviation. Data analysis was performed using the SPSS statistical software package (version 13.0; SPSS, Inc., Chicago, IL, USA). Differences between the experimental groups and control group were estimated using one-way analysis of variance (homogeneity of variances was $\mathrm{P}>0.05$ ) for the in vivo experiment, and analyzed using an independent-sample t-test for the in vitro experiment. $\mathrm{P}<0.05$ was considered to indicate a statistically significant difference.

\section{Results}

Astilbin relieve psoriasis-like lesions induced by propranolol chloride. Topical therapy is one of the most convenient methods for psoriasis. Therefore, astilbin was prepared into a microemulsion in the present study as a topical carrier for dermatological treatment, and its effect on guinea pigs was evaluated. The results showed that, following stimulation with propranolol chloride, the ears of the guinea pigs became red and swollen, accompanied by silvery scales and lesions, which remained until the end of experiment. However, the lesions on the animals treated with astilbin were milder, compared with those in the disease control group.

From the microscopic structure of the skin tissues, as shown as Fig. 2, the tissues in the disease control group exhibited parakeratosis and hyperkeratinization in the epidermis, thickened spinous layers and papillary papillae congestion in the derma; these histopathological characteristics are similar to those of psoriatic lesions in humans. By contrast, the astilbin microemulsion reduced the lesions in the cuticular layer, the granular layer appeared, and the spinous layers and derma decreased. Based on the Baker scores, the score of the disease control group was significantly increased, compared with that of the blank group. Topical administration of the astilbin microemulsion resulted in a significant decrease in the Baker score, as shown in Fig. 3, 
A

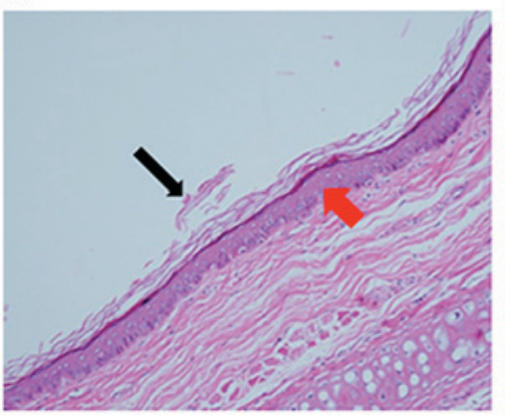

B

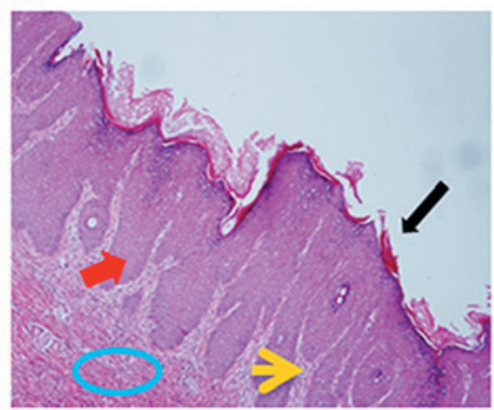

C

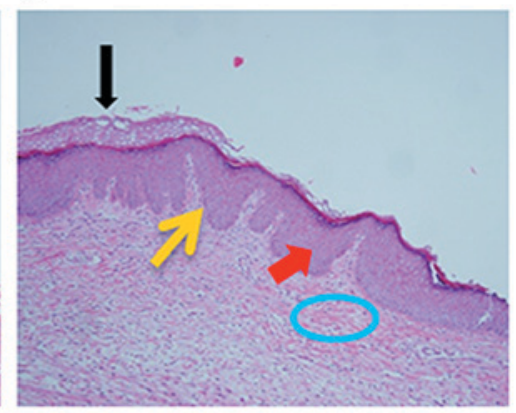

Figure 2. Histological sections of skin from the ears of guinea pigs (magnification, x100). (A) Blank group, in which skin was administered with vehicle cream. (B) Disease control group, in which propranolol hydrochloride cream was applied to skin with the vehicle cream. (C) Astilbin-treated group, in which propranolol hydrochloride cream was applied to skin with astilbin cream. Black arrows show the keratinized cuticular layers and granular layers, red arrows show the spinous layers, yellow arrows show lesions of derma and basal layer of epidermis, and the blue circle indicates inflammatory cells.

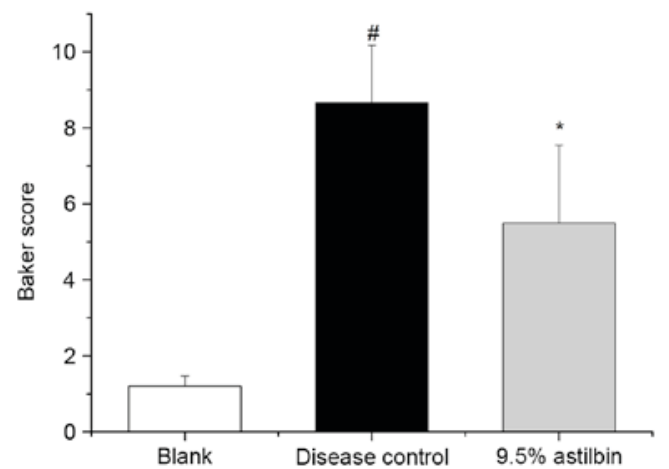

Figure 3. Baker scores of the lesions. ${ }^{\#} \mathrm{P}<0.05$ disease control vs. the blank control; ${ }^{\mathrm{P}}<0.059 .5 \%$ astilbin-treated group vs. the disease control. LPS, lipopolysaccharide.

with an inhibition ratio of $36.5 \%(\mathrm{P}<0.05)$. This suggested that the astilbin microemulsion ameliorated the negative effects in the psoriasis-like model in guinea pigs.

Astilbin has no effect on the viability of HaCaT cells. To investigate the effect of astilbin on $\mathrm{HaCaT}$ cell proliferation, an MTT method was used to detect the viability of $\mathrm{HaCaT}$ cells co-incubated with astilbin $(6.66,13.88,27.75,55.50$ and $222.00 \mu \mathrm{M}$ ). As shown in Fig. 4, no significant changes in the viability of the HaCaT cells were observed following co-incubation with astilbin at different concentrations for a 24-h incubation period. This result suggested that astilbin was non-toxic towards the HaCaT cells at the experimental dose range and may have no effect on the normal epidermis.

Astilbin inhibits the mRNA expression levels of IL-6, IL-17A and $I L-22$ in inflammation. In order to identify the cytokines targeted by astilbin in the inflamed skin microenvironment, the inflammatory model of HaCaT cells co-cultured with LPS for $2 \mathrm{~h}$ was established, and the mRNA expression levels of inflammatory cytokines associated with psoriasis, including IL-6, IL-4, IL-10 and IL-23 (some data not shown) were determined. The results showed that LPS increased the mRNA expression levels of IL-6, IL-17A and IL-22 in the HaCaT cells, whereas astilbin inhibited the mRNA expression levels of IL-6, IL-17A and IL-22 (Fig. 5). At a concentration of

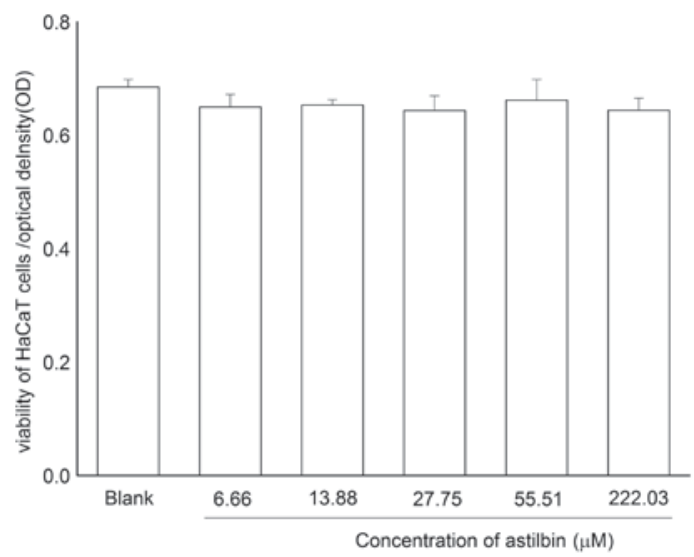

Figure 4. Effect of astilbin on the vitality of HaCaT cells. Results show cell viability at different concentrations of astilbin.

$2.22 \mu \mathrm{M}$, the inhibition ratio of astilbin was $89,69.1$ and $69.3 \%$ for IL-6, IL-17A and IL-22, respectively $(\mathrm{P}<0.01)$; however, at a concentration of $11.00 \mu \mathrm{M}$, astilbin only significantly decreased the mRNA expression of IL-6 (60.6\%; P<0.01), with no significant effects on IL-17A or IL-22.

Astilbin inhibits the protein expression levels of IL-6 and IL-22 stimulated by LPS in HaCaT cells. Whether changes in gene expression affect protein function depends on the subsequent protein translation. Therefore, the effects of astilbin on the protein expression levels of IL-6, IL-17A and IL-22 were detected in the same model using ELISA. As shown in Fig. 6, LPS increased the protein expression of IL- 6 and IL-22 by 36.0 and $46.5 \%$, respectively. Compared with the LPS-stimulated group, $2.22 \mu \mathrm{M}$ astilbin markedly decreased the expression levels of IL-6 and IL-22 by 79.2 and 49.5\%, respectively $(\mathrm{P}<0.05)$. The inhibition ratio of $11.10 \mu \mathrm{M}$ astilbin on IL-6 was $82.2 \%$. These levels were similar to the results of the RT-qPCR analysis. However, unlike the results of the RT-qPCR analysis, $11.10 \mu \mathrm{M}$ astilbin also significantly decreased the expression of IL-22 by $46.5 \%$. Neither LPS nor astilbin had a significant effect on the expression of IL-17A.

Astilbin inhibits the expression of phosphorylated p38 in HaCaT cells. MAPK signaling is an important signaling 


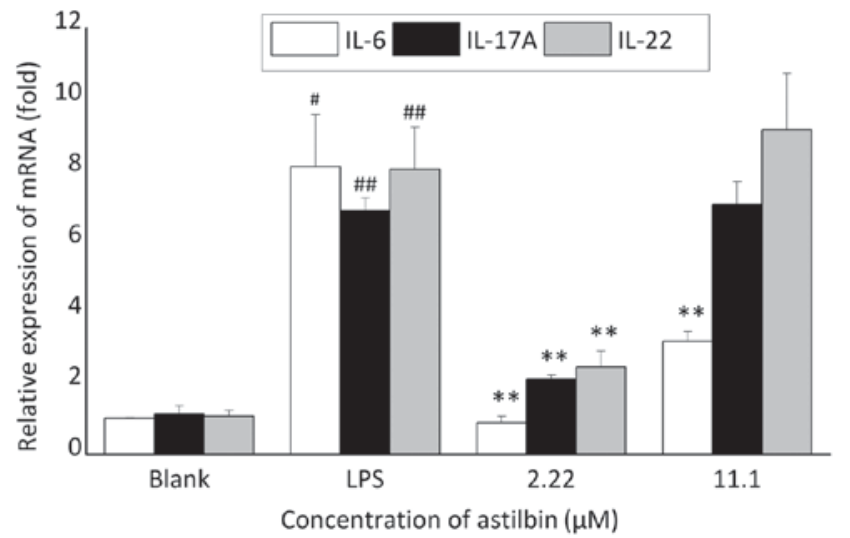

Figure 5. Effects of astilbin on mRNA expression levels of IL-6, IL-17A and IL-22. ${ }^{~} \mathrm{P}<0.05$, LPS group vs. blank control in IL-6 mRNA expression; ${ }^{\# \#} \mathrm{P}<0.01$, LPS group vs. blank control in IL-17A and IL-22 mRNA expression. ${ }^{* *} \mathrm{P}<0.01,2.22 \mu \mathrm{M}$ astilbin vs. LPS group in IL-6, IL-17A and IL-22 mRNA expression. ${ }^{* *} \mathrm{P}<0.01,11.1 \mu \mathrm{M}$ astilbin vs. LPS group in IL-6 mRNA expression. IL, interleukin; LPS, lipopolysaccharide.

pathway in psoriasis by regulating inflammation and proliferation. To examine whether astilbin inhibits IL-6 and IL-22 through MAPK signaling, the present study performed western blot analysis to analyze the expression of p38 MAPK and ERK1/2. As shown in Fig. 7, treatment with 2.22 and $11.10 \mu \mathrm{M}$ astilbin reduced the phosphorylation activity of p38 MAPK by 62.4 and $78.0 \%$, respectively $(\mathrm{P}<0.01)$ but had no significant effect on the phosphorylation of ERK1/2. These data support the hypothesis that astilbin targets p38 MAPK signaling to regulate downstream proteins, including inflammatory factors IL-6 and IL-22.

In order to confirm whether astilbin had a similar effect on the expression of IL- 6 and IL-22 with p38 MAPK inhibition, the present study used SB203580, a p38 MAPK inhibitor, for co-culture with the HaCaT cells prior to LPS treatment. As shown in Fig. 8, the p38 MAPK inhibitor suppressed the overexpression of IL-6 and IL-22 in the LPS-induced $\mathrm{HaCaT}$ cells, with inhibition ratios of 83.4 and $43.7 \%$, respectively $(\mathrm{P}<0.05)$, exhibiting the same effect as astilbin, which suggested that astilbin had a similar role as the p38 MAPK inhibitor in the inflammatory $\mathrm{HaCaT}$ cell model.

\section{Discussion}

Psoriasis is one of the most challenging diseases worldwide, which lacks complete treatment efficacy. For the majority of patients with mild-to-moderate psoriasis, topical treatment is the first option. Currently, there is an emphasis on the development of more effective drugs with fewer side effects for the treatment of psoriasis.

Astilbin is an active compound of the Chinese herb Smilax glabra Roxb. Accumulated evidence has shown that astilbin exerts varied activities, which may be associated with psoriasis (15). However, whether astilbin can be developed into a topical agent for psoriasis remains to be fully elucidated. In order to investigate this, the present study successfully prepared astilbin into a microemulsion and examined its effect on a psoriasis-like model in guinea pigs. The results showed that

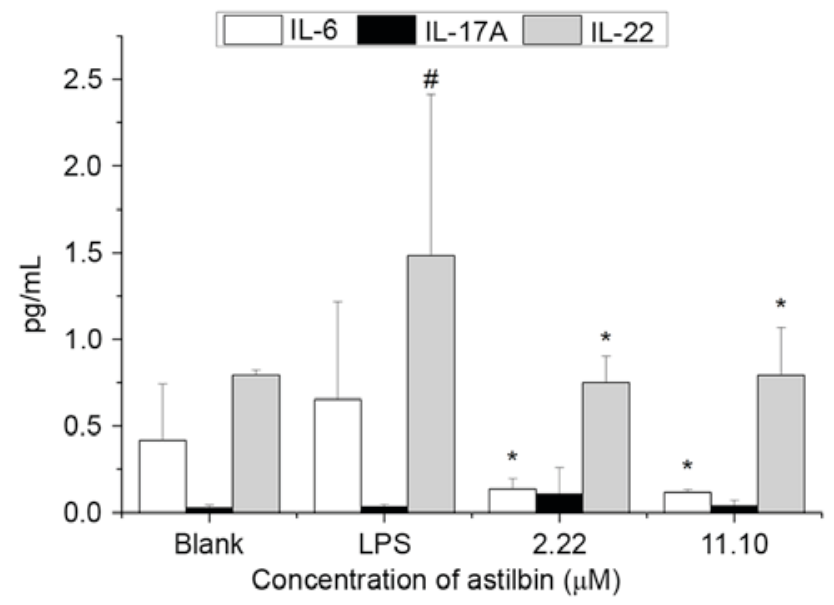

Figure 6. Secretion of IL-6, IL-17A and IL-22 in cells treated with astilbin. ${ }^{\#} \mathrm{P}<0.05$ LPS group vs. blank control; ${ }^{*} \mathrm{P}<0.052 .22$ and $11.1 \mu \mathrm{M}$ astilbin groups vs. the LPS group in IL-6 and IL-22 expression. IL, interleukin; LPS, lipopolysaccharide.

the topical administration of astilbin significantly ameliorated the psoriasis-like lesions stimulated by propranolol.

Based on the in vivo results of astilbin, the present study aimed to define the mechanism of astilbin in direct contact with inflamed or hyperplastic epidermal cells. Therefore, inflammatory HaCaT cells induced by LPS were used in the present study to examine how astilbin affected the epidermis cells. Astilbin was expected to suppress the proliferation of $\mathrm{HaCaT}$ cells, however, the results showed that astilbin had no effect on the proliferation of $\mathrm{HaCaT}$ cells, suggesting that anti-proliferative activity is not involved in the mechanism of astilbin.

Previous studies have indicated that astilbin has immunosuppressive activity in certain inflammatory diseases, including contact dermatitis, liver injury and colitis $(16,17)$, and that certain anti-inflammatory drugs, including glucocorticoids, have anti-psoriatic effects. This suggested that the anti-psoriatic effect of astilbin may be through anti-inflammatory effects. Cytokines are indicators of inflammation, and IL-6, IL-17A, IL-22, IL-23, IL-4 and IL-10 (18-20) are all upregulated in psoriatic lesions, a number of which have been used as a drug target and associated with disease severity. Therefore, the effect of astilbin on these cytokines was investigated in the present study. The results showed that astilbin decreased the expression of IL- 6 and IL-22 at the mRNA and protein levels, and inhibited the expression of IL-17A at the mRNA level only. Taken together, these results suggested that astilbin targeted IL- 6 and IL-22 to improve the microenvironment in the epidermis, rather than suppressing the normal proliferation of keratinocytes.

IL-6, IL-17 and IL-22 were all elevated in psoriatic skin. IL-6 is a multifunctional cytokine, which can be produced by keratinocytes in response to certain stimuli, including LPS (21). In psoriasis, IL-6 can directly promote epidermal hyperplasia and affect the function of dermal inflammatory cells (22). According to previous reports, astilbin decreased the serum cytokine level of IL-6 in lupus-prone mice and reduced the mRNA expression of IL-6 in LPS-stimulated J774A mice (23-25). The results of these studies are in accordance 


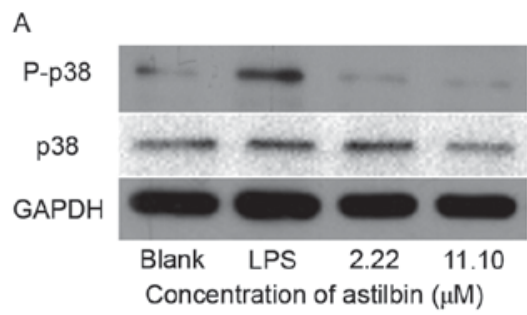

C

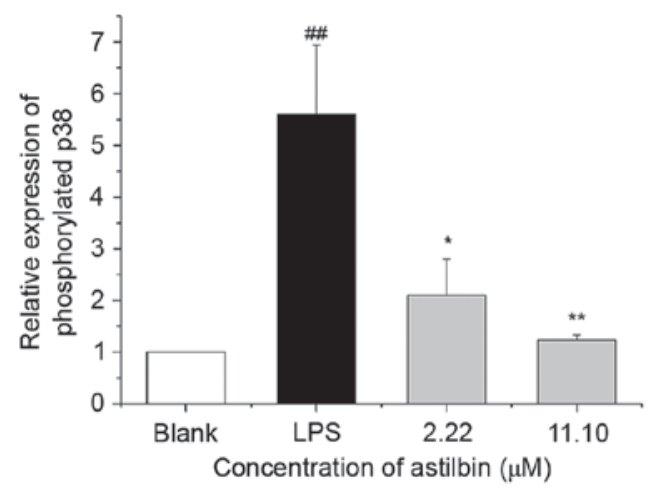

B

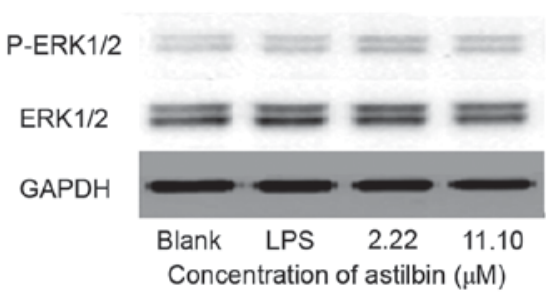

D

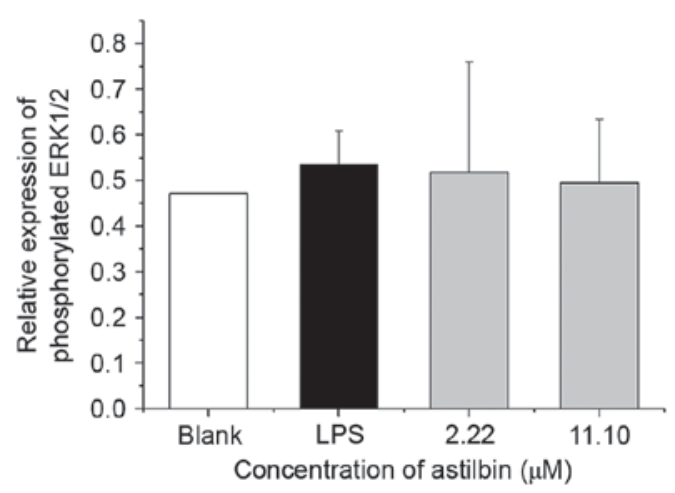

Figure 7. Effects of astilbin on expression levels of p-p38 and p-ERK1/2. The proteins were assessed using western blot analysis. Western blots analysis show (A) p-p38 MAPK and (B) p-ERK1/2. Graphs show quantification of the levels of (C) p-p38 MAPK and (D) p-ERK1/2. \#\# P<0.01 LPS group vs. the blank control; " $\mathrm{P}<0.05,2.22 \mu \mathrm{M}$ astilbin group vs. LPS group; ${ }^{* *} \mathrm{P}<0.0111 .1 \mu \mathrm{M}$ astilbin group vs. LPS group. MAPK, mitogen-activated protein kinase; ERK1/2, extracellular signal-regulated kinase 1/2; p, phosphorylated; LPS, lipopolysaccharide.

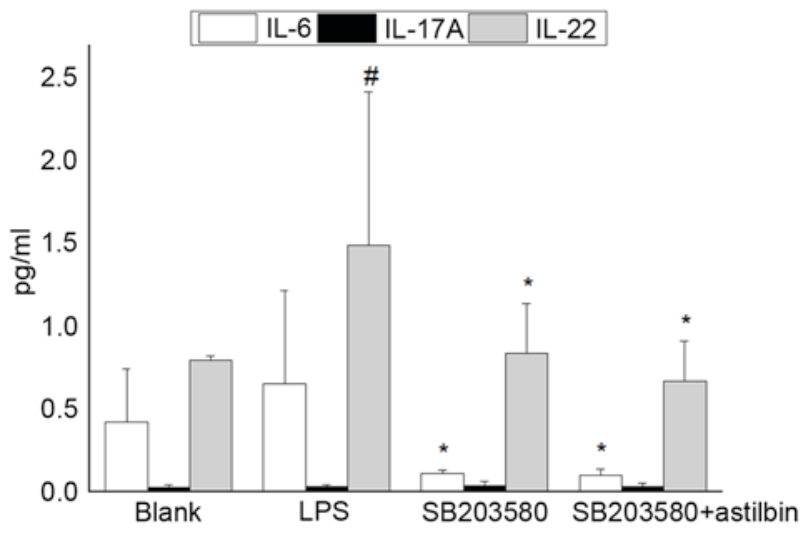

Figure 8. Effect of p38 MAPK inhibitor SB203580 on the protein expression of IL-6, IL-17A and IL-22. HaCaT cells were pretreated with SB203580 or a mixture of SB203580 and astilbin $(11.10 \mu \mathrm{M})$ for $30 \mathrm{~min}$, and then was incubated with LPS for $2 \mathrm{~h}$ prior to IL-6, IL-17A and IL-22 detection. ${ }^{\text {}} \mathrm{P}<0.05$ the LPS group vs. blank control in IL-22 expression; ${ }^{*} \mathrm{P}<0.05$ SB203580-treated or SB203580+astiblbin-treated group vs. LPS group in IL-6 and IL-22 expression. IL, interleukin; LPS, lipopolysaccharide.

with the present study and suggested that decreasing the expression of IL-6 is an anti-inflammatory function of astilbin, associated with the inhibition of hyperplasia and inflammatory infiltration in the psoriatic epidermis.

IL-22 was another cytokine targeted by astilbin in the present study. It has been reported that IL-22 induces features of psoriatic lesions, including inhibiting the differentiation and cornification of keratinocytes, disturbing the barrier function of the epidermis, facilitating immune cell infiltration and restructuring of the epidermis (26). Astilbin may have suppressed IL-22 to improve lesions in the psoriasis-like model. The association between astilbin and IL-22 in HaCaT cells has not been demonstrated previously. The downregulation of IL-22 may be a novel mechanism underlying the effect of astilbin on inflammatory diseases.

IL-17A is a pro-inflammatory cytokine, which has effects on neutrophil recruitment, host defense and immuno-inflammatory diseases, and is secreted predominantly by Th17 cells, mast cells, neutrophils and dendritic cells (27). Previous reports have indicated that astilbin decreases the serum cytokine level of IL-17A in lupus-prone mice (23). However, the present study showed that astilbin only marginally decreased the mRNA expression of IL-17A and had no effect on the protein expression of IL-17A. The reason for astilbin having different effects on mRNA and protein levels of IL-17A is unclear, however, it may be associated with the effect of LPS on the expression of IL-17A. Although there are reports of IL-17A responding to LPS-induced lung inflammation or other microbial infections $(28,29)$, these reports are based on in vivo experiments. In isolated epithelial cells, LPS or microbial infection may activate the gene activity of IL-17A and promote its transcription, however, the translation to protein depends on multiple factors, including the stability of mRNA, the abundance of specific codons and the longevity of proteins (30-32). Therefore, further investigations are required.

The expression of inflammatory cytokines are always regulated by a signaling molecule and, in order to identify the signaling pathway through which astilbin functions in the inflammatory model, p38 MAPK and EKR1/2 MAPK were selected for investigation. p38 MAPK and ERK1/2MAPK belong to MAPKs, and have been found to contribute to the pathogenesis of psoriasis. p38 MAPK inhibitors have been developed into potential biologicals for several inflammatory 
diseases, including arthritis and psoriasis $(33,34)$. Zou et al $(35)$ reported that astilbin suppressed the expression of p38 MAPK in an acute heart allograft rejection model, which was involved in inhibiting activated $\mathrm{T}$ cells. Consistent with this, the present study found that astilbin inhibited the phosphorylation of p38 MAPK in the HaCaT cells but had no effect on the phosphorylation of ERK1/2. This suggested that astilbin may suppress cytokines via p38 MAPK and ameliorate psoriasis. In order to investigate this, the present study used SB203580, a p38 MAPK inhibitor, for co-culture with the HaCaT cells prior to LPS exposure. The results showed that SB203580 inhibited the expression levels of IL-6 and IL-22 in the same manner as astilbin, which suggested that astilbin was involved as a p38 MAPK inhibitor in the inflamed HaCaT cell model.

In conclusion, the present study identified the anti-psoriatic activity of astilbin through the use of a psoriasis model in guinea pigs. The molecular mechanism involved the suppression of inflammatory cytokines IL-6 and IL-22 via the inhibition of p38 MAPK signaling. These results indicate that astilbin, as a natural micromolecule, offers potential for development as an effective drug to treat psoriasis by targeting p38 MAPK/IL-6/IL-22.

\section{Acknowledgements}

This study was supported by the Teamwork Project of the Natural Science Foundation of Guangdong Province of China (grant no. S2013030011515), the Project of the Traditional Chinese Medicine Hospital of Guangdong Province (grant no. YK2013B1N11), and the Project of the State Administration of Traditional Chinese Medicine Clinical Base Fund (grant no. JDZX2015206).

\section{References}

1. Abe R, Yamagishi S, Fujita Y, Hoshina D, Sasaki M, Nakamura K, Matsui T, Shimizu T, Bucala R and Shimizu H: Topical application of anti-angiogenic peptides based on pigment epithelium-derived factor can improve psoriasis. J Dermatol Sci 57: 183-191, 2010.

2. Richards HL, Fortune DG, O'Sullivan TM, Main CJ and Griffiths CE: Patients with psoriasis and their compliance with medication. J Am Acad Dermatol 41: 581-583, 1999.

3. Chen T, Li J, Cao J, Xu Q, Komatsu K and Namba T: A new flavanone isolated from Rhizoma Smilacis glabrae and the structural requirements of its derivatives for preventing immunological hepatocyte damage. Planta Med 65: 56-59, 1999

4. Guo J, Xu Q and Chen T: Quantitative determination of astilbin in rabbit plasma by liquid chromatography. J Chromatogr B Analyt Technol Biomed Life 805: 357-360, 2004.

5. Huang H, Cheng Z, Shi H, Xin W, Wang TT and Yu LL: Isolation and chacterization of two flavonoids, engeletin and astilbin from the leaves of Engelhardia roxburghiana and their potential anti-inflammatory properties. J Agric Food Chem 59: 4562-4569, 2011.

6. Bezerra GP, Góis RW, de Brito TS, de Lima FJ, Bandeira MA, Romero NR, Magalhães PJ and Santiago GM: Phytochemical study guided by the myorelaxant activity of the crude extract, fractions and constituent from stem bark of Hymeana courbaril L. J Ethnopharmacol 149: 62-69, 2013.

7. Yi HW, Lu XM, Fang F, Wang J and Xu Q: Astilbin inhibits the adhesion of $\mathrm{T}$ lymphocytes via decreasing TNF-alpha and its associated MMP-9 activity and CD44 expression. Int Immunopharmacol 18: 1467-1474, 2008.

8. Li GS, Jiang WL, Yue XD, Qu GW, Tian JW, Wu J and Fu FH: Effect of astilbin on experimental diabetic nephropathy in vivo and in vitro. Planta Med 75: 1470-1475, 2009.
9. Fei M, Wu X and Xu Q: Astilbin inhibits contact hypersensitivity through negative cytokine regulation distinct from cyclosporine A. J Allergy Clin Immunol 116: 1350-1356, 2005.

10. Zou S, Shen X, Tang Y, Fu Z, Zheng Q and Wang Q: Astilbin suppresses acute heart allograft rejection by inhibiting maturation and function of dendritic cells in mice. Transplant Proc 42: 3798-3802, 2010.

11. Guo L, Liu W, Lu T, Guo W, Gao J, Luo Q, Wu X, Sun Y, Wu X, Shen Y and Xu Q: Decrease of functional activated T and $\mathrm{B}$ cells and treatment of glomerulonephitis in Lupus-prone mice using a natural flavonid astilbin. PLoS One 10: e0124002, 2015.

12. Gaylarde PM, Brock AP and Sarkany I: Psoriasiform changes in guinea-pig skin from propranolol. Clin Exp Dermatol 3: 157-160, 1978.

13. Baker BS, Brent L, Valdimarsson H, Powles AV, al-Imara L, Walker M and Fry L: Is epidermal cell proliferation in psoriatic skin-grafts on nude-mice driven by T-cell derived cytokines. Br J Dermatol 12: 105-110, 1992.

14. Livak KJ and Schmittgen TD: Analysis of relative gene expression data using real-time quantitative PCR and the 2(T)(-Delta Delta C) method. Methods 25: 402-408, 2001.

15. Diao H, Kang Z, Han F and Jiang W: Astilbin protects diabetic rat heart against ischemia-reperfusion injury via blockade of HMGB1-dependent NF- $\kappa \mathrm{B}$ signaling pathway. Food Chem Toxicol 63: 104-110, 2014.

16. Fei MJ, Wu XF and Xu Q: Astilbin inhibits contact hypersensitivity through negative cytokine regulation distinct from cyclosporin A. J Allergy Clin Immunol 116: 1350-1356, 2005.

17. Ding Y, Liang Y, Deng B, Qiao A, Wu K, Xiao W and Gong W: Induction of TGF- $\beta$ and IL-10 production in dendritic cells using astilbin to inhibit dextran sulfate sodium-induced colitis. Biochem Biophys Res Commun 446: 529-534, 2014.

18. Puig L: Cardiovascular risk and psoriasis: The role of biologic therapy. Actas Dermosifiliogr 103: 853-862, 2012.

19. Menter A, Gottlieb A, Feldman SR, Van Voorhees AS, Leonardi CL, Gordon KB, Lebwohl M, Koo JY, Elmets CA, Korman NJ, et al: Guidelines of care for the management of psoriasis and psoriatic arthritis-section 1. Overview of psoriasis and guidelines of care for the treatment of psoriasis with biologics. J Am Acad Dermatol 58: 826-850, 2008.

20. Pietrzak AT, Zalewska A, Chodorowska G, Krasowska D, Michalak-Stoma A, Nockowski P, Osemlak P, Paszkowski T and Roliński JM: Cytokines and anticytokines in psoriasis. Clin Chim Acta 394: 7-21, 2008.

21. Jin J, Sundararaj KP, Samuvel DJ, Zhang X, Li Y, Lu Z, Lopes-Virella MF and Huang Y: Different signaling mechanisms regulating IL-6 expression by LPS between gingival fibroblasts and mononuclear cells: Seeking the common target. Clin Immunol 143: 188-199, 2012.

22. Grossman RM, Krueger J, Yourish D, Granelli-Piperno A, Murphy DP, May LT, Kupper TS, Sehgal PB and Gottlieb AB: Interleukin 6 is expressed in high levels in psoriatic skin and stimulates proliferation of cultured human keratinocytes. Proc Natl Acad Sci USA 86: 6367-6371, 1989.

23. Guo L, Liu W, Lu T, Guo W, Gao J, Luo Q, Wu X, Sun Y, Wu X, Shen Y and Xu Q: Decrease of functional activated T and B cells and treatment of glomerulonephitis in lupus-prone mice using a natural flavonoid astilbin. PLoS One 10: e0124002, 2015.

24. Huang H, Cheng Z, Shi H, Xin W, Wang TT and Yu L: Isolation and characterization of two flavonoids, engeletin and astilbin, from the leaves of engelhardia roxburghiana and their potential anti-inflammatory properties. J Agric Food Chem 59: 4562-4569, 2011.

25. Song SH, Shen XY, Liu F, Tang Y, Wang ZM and Fu ZR: Protective effects of astilbin on renal ischemia-reperfusion injury in rats. Zhong Xi Yi Jie He Xue Bao 7: 753-757, 2009 (In Chinese).

26. Hao JQ: Targeting Interleukin-22 in psoriasis. Inflammation 37: 94-99, 2014.

27. Iwakura Y, Nakae S, Saijo S and Ishigame H: The roles of IL-17A in inflammatory immune responses and host defense against pathogens. Immunol Rev 226: 57-79, 2008.

28. Grygorczuk S, Świerzbińska R, Moniuszko A, Kondrusik M, Zajkowska J, Czupryna P, Dunaj J and Pancewicz S: Synthesis of Th17 cytokines in the culture of peripheral blood mononuclear cells stimulated with Borrelia burgdorferi sensu lato. Ann Agric Environ Med 23: 242-247, 2016. 
29. Kim SR, Kim HJ, Kim DI, Lee KB, Park HJ, Jeong JS, Cho SH and Lee YC: Blockade of interplay between IL-17A and endoplasmic reticulum stress attenuates LPS-induced lung injury. Theranostics 5: 1343-1362, 2015.

30. Waggoner SA and Liebhaber SA: Regulation of alpha-globin mRNA stability. Exp Biol Med 228: 387-395, 2003.

31. Pesole G, Gissi C, Grillo G, Licciulli F, Liuni S and Saccone C: Analysis of oligonucleotide AUG start codon context in eukariotic mRNAs. Gene 261: 85-91, 2000.

32. Bachmair A, Finley D and Varshavsky A: In vivo half-life of a protein is a function of its amino-terminal residue. Science 234: 179-186, 1986
33. Mavropoulos A, Rigopoulou EI, Liaskos C, Bogdanos DP and Sakkas LI: The role of p38 MAPK in the aetiopathogenesis of psoriasis and psoriatic arthritis. Clin Dev Immunol 2013: 569751, 2013.

34. Johansen C, Kragballe K, Westergaard M, Henningsen J, Kristiansen $\mathrm{K}$ and Iversen L: The mitogen-activated protein kinases p38 and ERK1/2 are increased in lesional psoriatic skin. Br J Dermatol 152: 37-42, 2005.

35. Zou S, Shen X, Tang Y, Fu Z, Zheng Q and Wang Q: Astilbin suppresses acute heart allograft rejection by inhibiting maturation and function of dendritic cells in mice. Transplant Proc 42: 3798-3802, 2010. 